\title{
Fatores associados à desnutrição em idosos institucionalizados
}

\author{
Factors associated with malnutrition in institutionalized elderly
}

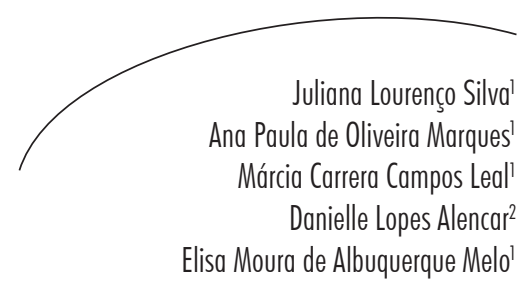

\section{Resumo}

Trata-se de artigo de revisão integrativa da literatura com o objetivo de analisar as evidências científicas que retratam os fatores associados à desnutrição em idosos institucionalizados. A busca de artigos foi realizada nas bases de dados LILACS, MEDLINE e IBECS, sendo incluídas as publicações referentes ao período de 2000 a novembro de 2012. Os artigos foram submetidos a dois instrumentos de avaliação, no intuito de verificar a qualidade metodológica dos estudos. Os resultados mostraram que os fatores psicológicos (depressão e demência) e funcionais (dependência) foram os principais aspectos relacionados à desnutrição, uma vez que a institucionalização favorece o isolamento e a inatividade física e mental, podendo comprometer a qualidade de vida do idoso. A desnutrição, apesar de associada ao aumento da morbi-mortalidade em geral, não é avaliada de forma rotineira nas instituições. Considerando as especificidades do segmento mais envelhecido, se faz necessário realizar avaliações multidimensionais da condição de saúde do idoso para possibilitar uma intervenção mais efetiva e integral.

\section{Abstract}

This literature review article aims to examine the scientific evidence that depict the associated factors with malnutrition in institutionalized elderly. The search for articles was conducted in the databases LILACS, MEDLINE and IBECS, including publications for the period 2000 to November 2012. The articles were also subjected to two assessment tools in order to verify the methodological quality of studies. The results showed that psychological factors (depression and dementia) and functional (dependence) were the main aspects related to malnutrition, since institutionalization fosters isolation and physical and mental inactivity, which may impair the quality of life of the elderly. Although malnutrition is associated with increased of morbidity and mortality, in general, it is not always assessed in institutions. Considering the specificities of the elderly segment, it is necessary to conduct multidimensional assessments of health status of the elderly to enable more effective and comprehensive interventions.

\footnotetext{
Universidade Federal de Pernambuco, Departamento de Medicina Social, Programa de Pós-graduação Integrado em Saúde Coletiva. Recife, PE, Brasil.

2 Universidade Federal de Pernambuco, Departamento de Enfermagem, Programa de Pós-graduação em Enfermagem. Recife, PE, Brasil.
}

Palavras-chave: Idoso. Instituição de Longa Permanência para Idosos. Desnutrição.

Key words: Elderly. Homes for the Aged. Malnutrition. 


\section{INTRODUÇÃO}

O envelhecimento populacional vem ocorrendo num contexto de importantes mudanças na sociedade. Nessa perspectiva, tem crescido a prestação de serviços de cuidados formais, entre os quais se situam as chamadas instituições de longa permanência para idosos (ILPI). Estas diferem dos hospitais e consistem em residências coletivas, com regras de convivência e lógica de operação que varia de acordo com seu público. Muitas vezes, cuidam de pessoas com fragilidades físicas e cognitivas em diversos graus de severidade; outras vezes oferecem apenas residência e abrigo. ${ }^{1}$

A institucionalização pode trazer inúmeras consequências, tanto positivas quanto negativas, como reflexos da adaptação à vida asilar. A mudança para a instituição pode significar para o idoso uma espécie de confinamento, uma vez que esse se vê muitas vezes privado de suas atividades familiares e rotineiras, sendo obrigado a viver uma situação limitada e prejudicada, podendo comprometer sua qualidade de vida. ${ }^{2}$

O envelhecimento, apesar de ser um processo natural, submete o organismo a diversas alterações anatômicas e funcionais, com repercussões nas condições de saúde e nutrição do idoso. ${ }^{3}$ Além dos condicionantes específicos do próprio envelhecimento, existem outros fatores que podem afetar o estado nutricional dessa população, tais como: situação social (pobreza, isolamento social), alterações psicológicas (demência, depressão), condição de saúde (doenças crônicas, disfagia, polifarmácia, alterações na mastigação, perda da capacidade funcional e autonomia), entre outros. ${ }^{4}$

As consequências dos fatores de risco acima mencionados estão muitas vezes associadas ao menor consumo alimentar, tornando os idosos vulneráveis do ponto de vista nutricional. O desequilíbrio nutricional no idoso está relacionado positivamente ao aumento da morbimortalidade, à susceptibilidade a infecções e à redução da qualidade de vida. ${ }^{5}$ Nos últimos anos, os estudos mostram alta prevalência de idosos desnutridos. Os valores oscilam de 15 a
$60 \%$, dependendo do local onde o idoso vive em casa, asilo ou hospital - e da técnica utilizada para o diagnóstico de desnutrição. ${ }^{6}$

Considerando o grau de vulnerabilidade que os idosos residentes em ILPI apresentam, tanto pelo aspecto relacionado à idade, quanto pelas condições impostas pela institucionalização, verifica-se a necessidade de realizar estudos que retratem a magnitude da desnutrição na população geriátrica, principalmente no que diz respeito aos fatores associados a essa condição.

Nessa perspectiva, o presente estudo se propôs a analisar as evidências científicas que retratam os fatores associados à desnutrição em idosos institucionalizados, no intuito de contribuir para uma assistência mais integral e qualificada, possibilitando assim melhor qualidade de vida para esses idosos.

\section{METODOLOGIA}

Trata-se de uma revisão integrativa que possui a finalidade de reunir e sintetizar resultados de pesquisas sobre uma questão delimitada, de maneira sistemática e ordenada, contribuindo para o aprofundamento do conhecimento do tema investigado. Esse tipo de revisão permite apontar lacunas do conhecimento que precisam ser preenchidas com a realização de novos estudos.?

Para o desenvolvimento da presente revisão integrativa, as seguintes etapas foram percorridas: elaboração da questão norteadora; estabelecimento de critérios de inclusão e exclusão de artigos; definição das informações a serem extraídas dos artigos selecionados; avaliação dos estudos incluídos; interpretações dos resultados e apresentação da revisão integrativa. ${ }^{8}$ Para orientar o estudo, formulou-se a seguinte questão: quais as evidências científicas que retratam os fatores associados à desnutrição em idosos institucionalizados?

Os critérios de elegibilidade para inclusão no estudo foram: texto completo disponível online; artigos publicados no período de 2000 a novembro de 2012; divulgados em língua 
portuguesa, espanhola ou inglesa, e cujas publicações considerassem pessoa idosa aquela com 60 anos de idade ou mais. Descartaram-se publicações referentes a revisão bibliográfica e artigos repetidos em uma ou mais bases de dados.

A coleta de dados ocorreu durante o mês de novembro de 2012, e para a seleção dos artigos utilizaram-se as seguintes bases de dados eletrônicas: Literatura Latino-Americana em Ciências da Saúde (LILACS), Medical Literature and Retrieval System Online (MEDLINE) e Índice Bibliográfico Español em Ciencias de la Salud (IBECS).

$\mathrm{Na}$ busca de artigos, foram empregados os descritores padronizados pelo Descritores em Ciências da Saúde (DeCS): idoso, desnutrição e instituição de longa permanência para idosos. Foi realizado cruzamento entre os descritores por meio da estratégia de pesquisa combinada com o conector "AND" no campo "descritor de assunto" (tabela 1).

Tabela 1. Descritores utilizados na busca de artigos para revisão integrativa. Recife-PE, 2012.

\begin{tabular}{lll}
\hline \multicolumn{1}{c}{ Português } & \multicolumn{1}{c}{ Inglês } & \multicolumn{1}{c}{ Espanhol } \\
\hline Idoso + Desnutrição & Aged + Malnutrition & Anciano + Desnutrición \\
$\begin{array}{l}\text { Idoso + Instituição de Longa } \\
\text { Permanência para Idosos }\end{array}$ & Aged + Homes for the Aged & Anciano + Hogares para Ancianos \\
$\begin{array}{l}\text { Desnutrição + Instituição de Longa } \\
\text { Permanência para Idosos }\end{array}$ & Malnutrition + Homes for the & Desnutrición + Hogares para \\
Idoso + Desnutrição + Instituição de & Aged & Ancianos \\
Longa Permanência para Idosos & for the Aged & Anciano + Desnutrición + \\
\hline
\end{tabular}

Após a busca, foi realizada a pré-seleção dos artigos por meio da leitura criteriosa dos títulos e resumos, no intuito de verificar a adequação dos mesmos aos critérios estabelecidos. Após o levantamento dos artigos, os estudos foram submetidos a dois instrumentos para avaliação da qualidade metodológica: adaptado do Critical Apppraisal Skills Programme (CASP) ${ }^{9}$ e Classificação Hierárquica das Evidências para Avaliação dos Estudos.

O CASP é composto por dez itens (máximo 10 pontos), abrangendo: 1) objetivo; 2) adequação metodológica; 3) apresentação dos procedimentos teóricos e metodológicos; 4) seleção da amostra; 5) procedimento para a coleta de dados; 6) relação entre o pesquisador e pesquisados; 7) consideração dos aspectos éticos; 8) procedimento para a análise dos dados; 9) apresentação dos resultados; 10) importância da pesquisa. Os estudos foram classificados de acordo com as seguintes pontuações: 6 a 10 pontos (boa qualidade metodológica e viés reduzido), e mínima de 5 pontos (qualidade metodológica satisfatória, porém com risco de viés aumentado). Neste estudo, optou-se por utilizar apenas os artigos classificados de 6 a 10 pontos.

$\mathrm{O}$ segundo instrumento corresponde à Classificação Hierárquica das Evidências para Avaliação dos Estudos, ${ }^{10}$ que contempla os seguintes níveis: 1) revisão sistemática ou metánalise; 2) ensaios clínicos randomizados; 3 ) ensaio clínico sem randomização; 4) estudos de coorte e de caso-controle; 5) revisão sistemática de estudos descritivos e qualitativos; 6) único estudo descritivo ou qualitativo; 7) opinião de autoridades e/ou relatório de comitês de especialidades. Na avaliação, optou-se por não utilizar artigos com nível 7.

\section{RESULTADOS}

A figura 1 apresenta os resultados encontrados com base na busca pelos descritores, conforme cada base de dados. 


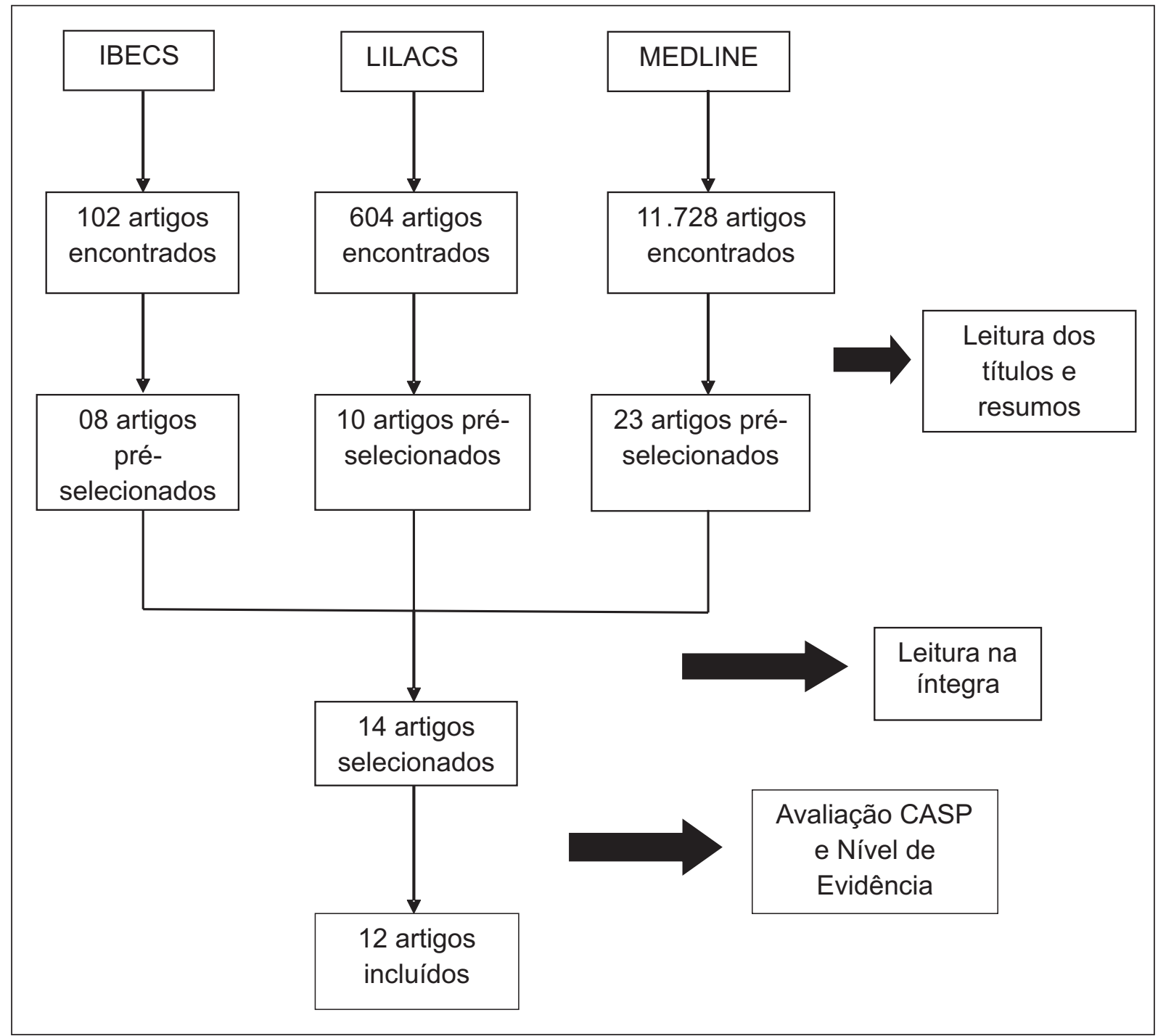

Figura 1. Diagrama dos artigos encontrados e selecionados por meio da pesquisa nas bases de dados. Recife-PE, 2012.

A maioria das pesquisas foi realizada em países europeus, como Alemanha, França e Espanha. Também foram identificados estudos feitos no Brasil, Canadá, Egito e Taiwan. Na tabela 2, encontra-se a caracterização dos artigos incluídos na presente revisão integrativa. 
Tabela 2. Caracterização dos artigos incluídos na revisão integrativa. Recife-PE, 2012.

\begin{tabular}{|c|c|c|c|c|}
\hline Título & Autor & Periódico & Ano & Base de dados \\
\hline $\begin{array}{l}\text { Nutrition status among residents living in a } \\
\text { veterans' long-term care facility in Western } \\
\text { Canada: a pilot study }\end{array}$ & Boström AM. et al. ${ }^{4}$ & $\begin{array}{l}J \text { Am Med Dir } \\
\text { Assoc. }\end{array}$ & 2011 & MEDLINE \\
\hline $\begin{array}{l}\text { Undernutrition in geriatric institutions in } \\
\text { South-West France: policies and risk factors }\end{array}$ & $\begin{array}{l}\text { Bourdel-Marchasson } \\
\text { I. et al. } .^{19}\end{array}$ & Nutrition & 2009 & MEDLINE \\
\hline $\begin{array}{l}\text { Asociación entre riesgo de desnutrición } \\
\text { y capacidad afectiva en personas de edad } \\
\text { avanzada institucionalizadas de la } \\
\text { Comunidad de Madrid }\end{array}$ & Centurión L. et al. ${ }^{13}$ & $\begin{array}{l}\text { Nutr. clin. diet. } \\
\text { Hosp. }\end{array}$ & 2010 & IBECS \\
\hline $\begin{array}{l}\text { Prevalence and factors associated with } \\
\text { feeding difficulty in institutionalized elderly } \\
\text { with dementia in Taiwan }\end{array}$ & Chang CC..$^{14}$ & $\begin{array}{l}\text { J Nutr Health } \\
\text { Aging }\end{array}$ & 2012 & MEDLINE \\
\hline $\begin{array}{l}\text { Correction of nutrition test errors for more } \\
\text { accurate quantification of the link between } \\
\text { dental bealth and malnutrition }\end{array}$ & Dion N. et al. ${ }^{15}$ & Nutrition & 2007 & MEDLINE \\
\hline $\begin{array}{l}\text { Aspectos sociodemográficos, de saúde e nivel } \\
\text { de satisfação de idosos institucionalizados } \\
\text { no Distrito Federal }\end{array}$ & Araújo N. et al. ${ }^{29}$ & Rev. ciênc. méd. & 2008 & LILACS \\
\hline $\begin{array}{l}\text { Nutritional status in older adults with mild } \\
\text { cognitive impairment living in elderly homes } \\
\text { in Cairo, Egypt }\end{array}$ & $\begin{array}{l}\text { Khater MS. \& } \\
\text { Abouelezz NF. }\end{array}$ & $\begin{array}{l}\text { J Nutr Health } \\
\text { Aging }\end{array}$ & 2011 & MEDLINE \\
\hline $\begin{array}{l}\text { Malnutrition and depression in the } \\
\text { institutionalised elderly }\end{array}$ & Smoliner C. et al. ${ }^{17}$ & BrJ Nutr. & 2009 & MEDLINE \\
\hline $\begin{array}{l}\text { Care problems and nursing interventions } \\
\text { related to oral intake in German nursing } \\
\text { homes and hospitals: a descriptive } \\
\text { multicenter study }\end{array}$ & Tannen A. et al. ${ }^{20}$ & Int J Nurs Stud. & 2012 & MEDLINE \\
\hline $\begin{array}{l}\text { Risk of malnutrition among Brazilian } \\
\text { institutionalized elderly: a study with the } \\
\text { Mini Nutritional Assessment (MNA) } \\
\text { questionnaire }\end{array}$ & $\begin{array}{l}\text { Pereira MRS. \& Santa } \\
\text { Cruz CMA. }{ }^{30}\end{array}$ & $\begin{array}{l}\text { J Nutr Health } \\
\text { Aging }\end{array}$ & 2011 & MEDLINE \\
\hline $\begin{array}{l}\text { The impact of self-perceived masticatory } \\
\text { function on nutrition and gastrointestinal } \\
\text { complaints in the elderly }\end{array}$ & $\begin{array}{l}\text { Altenhoevel A. et } \\
\text { al. }^{24}\end{array}$ & $\begin{array}{l}\text { J Nutr Health } \\
\text { Aging }\end{array}$ & 2012 & MEDLINE \\
\hline $\begin{array}{l}\text { Estado nutricional de idosos em uma } \\
\text { instituição de longa permanência }\end{array}$ & Lehn F. et al. ${ }^{25}$ & $\begin{array}{l}\text { J. Health Sci. } \\
\text { Inst. }\end{array}$ & 2012 & LILACS \\
\hline
\end{tabular}


Em relação ao tipo de periódico, oito artigos foram publicados em revistas da área de Nutrição, dois em revista médica, um na área de Enfermagem e um em Ciências da Saúde. Do total de publicações, oito foram desenvolvidos em universidades, e ainda foi encontrada uma pesquisa multicêntrica; já em outra publicação, não foi possível identificar a instituição sede do estudo.
Quanto às características metodológicas, todos os artigos foram de abordagem quantitativa e apresentaram delineamento não experimental, do tipo transversal. Dessa forma, os 12 artigos analisados apresentaram nível de evidência 6 . $\mathrm{Na}$ tabela 3, apresenta-se a síntese dos artigos incluídos na revisão integrativa.

Tabela 3. Apresentação da síntese de artigos incluídos na revisão integrativa. Recife-PE, 2012.

\begin{tabular}{|c|c|}
\hline Parâmetros & Síntese \\
\hline $\begin{array}{l}\text { Métodos de avaliação } \\
\text { nutricional }\end{array}$ & $\begin{array}{l}\text { Antopometria }{ }^{13,14,20,24,25,30} \text {,Consumo Alimentar }{ }^{14} \text {, Avaliação Subjetiva } \\
\text { Global }{ }^{4,13,15-17,19,24,29,30} \text {. }\end{array}$ \\
\hline Fatores associados & $\begin{array}{l}\text { Sexo feminino }{ }^{25,29} \text {, idosos com idade avançada } a^{30} \text {, baixa renda }{ }^{30} \text {, menor nível de } \\
\text { escolaridade }{ }^{30} \text {, depressã } 0^{4,13,15,17} \text {, demência }{ }^{4,14,16} \text {, comprometimento cognitivo }{ }^{14} \text {, } \\
\text { maior grau de dependência }{ }^{14,15,19,20} \text {, distúrbios de deglutição } 0^{15,20} \text {, capacidade } \\
\text { mastigatória reduzida }{ }^{14,20,24,25} \text {, perda de apetite }{ }^{20} \text {, náuseas }{ }^{20} \text {. }\end{array}$ \\
\hline
\end{tabular}

Instrumentos de medida Miniavaliação Nutricional $1^{4,13,15,16,17,19,24,29,30}$, Assessment Instrument-Minimum Data Set - RAI-MDS 2.0 $0^{4}$, Miniexame do Estado Mental ${ }^{13,16}$, Escala de Depressão Geriátrica ${ }^{13,16,17,24}$, Short Portable Mental Status Questionnaire-SPMSQ ${ }^{14}$, Montreal Cognitive Assessment - MoCA ${ }^{16}$, Edinburgh Feeding Evaluation in Dementia - EdFED Scale ${ }^{14}$, Índice de Barthel ${ }^{13,14,17,24}$, Groupe Iso-Ressource - GIR instrument ${ }^{15}$, Care Dependency Scale - $\mathrm{CDS}^{20}$.

Variáveis investigadas

Estado nutricional ${ }^{4,13,14-17,19,20,24,25,29,30}$, idade e sexo ${ }^{4,13-17,20,24,25,29,30}$, estado civil e escolaridade $^{13-16,29,30}$, renda ${ }^{15,30}$, naturalidade ${ }^{29}$, profissã $0^{13,15,29}$, condição de saúde (tipo e número de doenças e medicamentos em uso) ${ }^{4,13-17,20,24,25,29}$, presença de depressão $0^{4,13,17,24}$,capacidade funcional ${ }^{13,14,16,17,19,24,29}$, função cognitiva ${ }^{4},{ }^{13,14,16,19,29}$, depedência ${ }^{15,20}$, capacidade mastigatória ${ }^{15,20,24}$, dificuldade na ingestão alimentar ${ }^{14,19,20}$, função gastrointestinal ${ }^{24}$, características das instituições (tipo e natureza) $)^{15,19}$, tempo de institucionalização ${ }^{4,15,25}$, satisfação com as instituições ${ }^{29}$, saúde oral ${ }^{15,24,25}$, qualidade de vida ${ }^{17}$, hábitos de vida ${ }^{29}$, acuidade visual e auditiva ${ }^{29}$, visitas de familiares e amigos ${ }^{29}$, plano de cuidado/assistência especializada ${ }^{15,20}$.

Tamanho da amostra (quantitativo de indivíduos com idade $\geq 60$ anos)

Limitações do estudo
Conveniência: $112^{24}, 187^{29}, 55^{4}, 68^{13}, 93^{14}, 114^{17}, 5.521^{20}, 344^{30}, 42^{25}$.

Randômica: $601^{19}, 1.904^{15}, 120^{16}$.

Amostra reduzida (inviabilizando generalizações) ${ }^{4,13,14,25}$, seleção de amostra por conveniência (não descartando possibilidades de vieses) ${ }^{17,20,24,30}$, coleta de dados (informação obtida por terceiros - cuidador formal e/ou idoso sem avaliação prévia de cognição) ${ }^{14,20,29}$, amostra predominantemente masculina ${ }^{4}$, não identificado (apesar de referido pelos autores, não devidamente explicitado) $)^{15,16,19}$. 


\section{DISCUSSÃO}

O envelhecimento populacional vem ocorrendo em um contexto de grandes mudanças sociais, culturais e econômicas. Embora as políticas priorizem a família como responsável pelo cuidado dos membros dependentes, isso se torna cada vez mais escasso, em função da redução da fecundidade, das mudanças no arranjo familiar e da crescente participação da mulher - tradicional cuidadora - no mercado de trabalho. ${ }^{11}$ Dessa forma, vem ocorrendo o aumento da demanda por ILPIs que, em muitos casos, se torna importante alternativa no cuidado ao idoso.

Embora as ILPIs atendam aos idosos quanto às necessidades de moradia, higiene, alimentação e acompanhamento médico, há o inconveniente de afastar o idoso de seu convívio familiar, favorecendo o isolamento e a inatividade física e mental, com consequências negativas em sua qualidade de vida. ${ }^{12}$ Nesse contexto, a condição de déficit nutricional torna-se mais alarmante, pois não somente os fatores relacionados ao envelhecimento irão influenciar o estado de nutrição do idoso, mas o próprio ambiente asilar representa importante fator de risco.

Nessa perspectiva, a maioria dos estudos da presente revisão integrativa associou as condições neuropsicológicas como importantes fatores para o desenvolvimento da desnutrição em idosos institucionalizados. ${ }^{13-17}$ A relação causal entre depressão e desnutrição ainda é incerta. Tem sido observado que os indivíduos deprimidos têm, frequentemente, maiores deficiências de algumas vitaminas, tais como ácido fólico ou piridoxina, e alguns minerais, como magnésio ou zinco, em comparação com indivíduos sem depressão. Além disso, a depressão tem sido identificada como uma das principais causas de perda de peso em pessoas idosas. ${ }^{13}$

Cabe ainda destacar que idosos demenciados passam por diversas fases de alterações cognitivas e, conforme a evolução do quadro, muitas vezes as necessidades básicas passam despercebidas pelos idosos, tais como sede e fome. ${ }^{18}$ Além disso, pessoas com demência apresentam muita dificuldade durante o ato de se alimentar, como manter a boca fechada, atraso na deglutição e voltando a cabeça para trás durante a alimentação. ${ }^{14}$

No que se refere à capacidade funcional, os estudos verificaram que idosos desnutridos apresentaram maior dependência nas atividades de vida diária, especialmente as relacionadas ao modo de se alimentar. ${ }^{14,15,19,20}$ Muitos não conseguiam se alimentar sozinhos e precisavam de apoio no momento das refeições, e ainda necessitavam de certas adaptações dos talheres e outros utensílios para facilitar a alimentação.

Segundo Caldas, ${ }^{21}$ o processo de envelhecimento, por si só, pode acarretar o declínio da aptidão física e da capacidade funcional, que se agrava com o sedentarismo, tornando os idosos dependentes de cuidados de outrem. Observa-se que tal situação prevalece mais entre os idosos institucionalizados, tornando-os detentores de várias consequências decorrentes da inatividade..$^{22}$ Destaca-se, ainda, que a associação do envelhecimento fisiológico com doenças crônico-degenerativas, bastante prevalentes em idosos, torna essa faixa etária muito vulnerável à deterioração físico-funcional, com consequente perda de autonomia e independência. ${ }^{23}$

Em relação à saúde oral, alguns artigos identificaram o edentulismo, perda dentária parcial e distúrbios de deglutição (como a disfagia) como importantes fatores de risco para a condição nutricional do idoso, ${ }^{15,20,24,25} \mathrm{em}$ função de prejuízos no processo de mastigação, dificultando a digestão e levando à restrição alimentar. Nessa perspectiva, os estudos demonstraram a importância de uma melhor adequação da dieta, conforme a condição individual do idoso, tanto no aspecto da consistência dos alimentos, quanto ao modo de se alimentar (ex.: postura ereta, comer devagar).

Embora os resultados da presente revisão integrativa sejam importantes para o conhecimento dos fatores associados à desnutrição, esses devem ser vistos com cautela, pois todos os artigos analisados foram de 
corte transversal e, segundo Almeida Filho \& Rouquayrol, ${ }^{26}$ esse tipo de estudo epidemiológico é puramente descritivo e limita-se apenas à testagem de hipóteses de associação, sendo de baixo poder analítico.

No que se refere aos instrumentos de medida utilizados nos estudos, merece destaque a Miniavaliação Nutricional (MAN) que representa um método de avaliação nutricional subjetiva, considerado como de alta sensibilidade e especificidade na identificação de risco nutricional e desnutrição em idosos. ${ }^{27}$ Além disso, observou-se a importância da aplicação de outros instrumentos de avaliação para que melhor retratassem os fatores associados à desnutrição em idosos institucionalizados, como a Escala de Depressão Geriátrica, Índice de Barthel e Miniexame do Estado Mental.

\section{CONCLUSÃO}

$\mathrm{Na}$ presente revisão integrativa, as condições impostas pela institucionalização, como isola- mento e limitações das atividades de vida diária, são importantes fatores para a condição de saúde e nutrição de idosos asilados. Ainda que em caráter hipotético, dada a natureza transversal dos estudos, esses fatores podem influenciar o aspecto cognitivo do idoso, predispondo à depressão e outros problemas psíquicos, os quais favorecem mudanças no comportamento alimentar, com baixa ingestão de alimentos e maior ocorrência de desnutrição.

A desnutrição, apesar de associada ao aumento da morbi-mortalidade, perda da autonomia e redução da qualidade de vida, ${ }^{28-30} \mathrm{em}$ geral não é avaliada de forma rotineira nas instituições de longa permanência para idosos. A insuficiência de estudos nacionais que tenham por casuística idosos institucionalizados dificulta a magnitude do problema.

Considerando as especificidades do segmento mais envelhecido, se faz necessário realizar avaliações multidimensionais da condição de saúde do idoso para possibilitar uma intervenção mais efetiva e integral.

\section{REFERÊNCIAS}

1. Galesi LF, Lorenzetti C, Oliveira MRM, Fogaça KCP, Merhi VL. Perfil alimentar e nutricional de idosos residentes em moradias individuais numa instituição de longa permanência no leste do estado de São Paulo. Rev Aliment Nutr 2008;19(3):283-90.

2. Pestana LC, Espírito Santo FH. As engrenagens da saúde na terceira idade: um estudo com idosos asilados. Rev Esc Enferm USP 2008;42(2):268-75.

3. Pfrimer K, Ferriolli E. Fatores que interferem no estado nutricional do idoso. In: Vitolo MR, editor. Nutrição: da gestação ao envelhecimento. Rio de Janeiro: Rubio; 2008. p. 459-65.

4. Boström AM, Soest DV, Kolewaski B, Milke DL, Estabrooks CA. Nutrition status among residents living in a veterans' long-term care facility in Western Canada: a pilot study. J Am Med Dir Assoc 2011;12(3):217-25.

5. Felix LN, Souza EMT. Avaliação nutricional de idosos em uma instituição por diferentes instrumentos. Rev Nutr 2009;22(4):571-80.

6. Casas JR, Pinyol Martínez M, De Pedro Elvira B, Devant Altimir M, Benavides Ruiz A. Desnutrición en pacientes en atención domiciliaria. Aten Prim 2004;34(5):238-43.

7. Mendes KDS, Silveira RCCP, Galvão CM. Revisão integrativa: método de pesquisa para a incorporação de evidências na saúde e na enfermagem. Texto \& Contexto Enferm 2008;17(4):758-64.

8. Beya S, Nicoll LH. Writing an integrative review. AORN J 1998;67(4):877-80.

9. Milton Keynes Primary care trust. Critical Appraisal Skills Programme. London: Oxford; 2002.

10. Stillwell SB, Fineout-Overholt E, Melnyk BM, Williamson KM. Evidence-based practice: step by step. Am J Nurs 2010;110(1):51-3.

11. Camarano AA, Kanso S. As instituições de longa permanência para idosos no Brasil. Rev Bras Estud Popul 2010;27(1):233-5. 
12. Herédia VBM, Casara MB, Cortelletti IA, Ramalho $\mathrm{MH}$, Sassi A, Borges MN. A Realidade do Idoso Institucionalizado. Textos Envelhecimento 2004;7(2):9-31.

13. Centurión L, Aparicio VA, Ortega ARM, RodríguezRodríguez E, López-Plaza B, López-Sobaler AM. Asociación entre riesgo de desnutrición y capacidad afectiva en personas de edad avanzada institucionalizadas de la Comunidad de Madrid. Nutr Clín Diet Hosp 2010;30(2):33-41.

14. Chang CC. Prevalence and factors associated with feeding difficulty in institutionalized elderly with dementia in Taiwan. J Nutr Health Aging 2012; 16(3):258-61.

15. Dion N, Cotart JL, Rabilloud M. Correction of nutrition test errors for more accurate quantification of the link between dental health and malnutrition. Nutrition 2007;23(4):301-7.

16. Khater MS, Abouelezz NF. Nutritional status in older adults with mild cognitive impairment living in elderly homes in Cairo, Egypt. J Nutr Health Aging 2011;15(2):104-8.

17. Smoliner C, Norman K, Wagner KH, Harting W, Lochs H, Pirlich M. Malnutrition and depression in the institutionalised elderly. Br J Nutr 2009;102(7):1663-7.

18. Castro PR, Frank AA. Mini Avaliação nutricional na determinação do estado de saúde de idosos com ou sem a doença de Alzheimer: aspectos positivos e negativos. Estud Interdiscip Envelhec 2009;14(1):45-64.

19. Bourdel-Marchasson I, Rolland C, Jutand MA, Egea C, Baratchart B, Barberger-Gateau P. Undernutrition in geriatric institutions in South-West France: policies and risk factors. Nutrition 2009;25(2):155-64.

20. Tannen A, Schutz T, Smoliner C, Dassen T, Lahmann $\mathrm{N}$. Care problems and nursing interventions related to oral intake in German nursing homes and hospitals: a descriptive multicenter study. Int J Nurs Stud 2012;49(4):378-85.
21. Caldas CP. Envelhecimento com dependência: responsabilidades e demandas da família. Cad Saúde Pública 2003;19(3):733-81.

22. Gonçalves LHT, Silva AH, Mazo GZ, Benedetti TRB, Santos SMA, Marques S, et al. O idoso institucionalizado: avaliação da capacidade funcional e aptidão física. Cad Saúde Pública 2010;26(9): 1738-46.

23. Paschoal SMP, Salles RFN, Franco RP. Epidemiologia do envelhecimento. In: Carvalho ET Filho, Papaléo Netto M, editores. Geriatria: fundamentos, clínica e terapêutica. São Paulo: Atheneu; 2005: p. 19-35.

24. Altenhoevel A, Norman K, Smoliner C, Peroz I. The impact of self-perceived masticatory function on nutrition and gastrointestinal complaints in the elderly. J Nutr Health Aging 2012;16(2):175-8.

25. Lehn F, Coelho HDS, Garcia MT, Scabar LF. Estado nutricional de idosos em uma instituição de longa permanência. J Health Sci Inst 2012;30(1):53-8.

26. Almeida N Filho, Rouquayrol MZ. Elementos de metodologia epidemiológica. In: Rouquayrol MZ, editor. Epidemiologia e saúde. Rio de Janeiro: Medsi; 2003. p. $149-77$.

27. Guigoz Y, Lauque S, Vellas BJ. Identifying the elderly at risk for malnutrition: the mini nutritional assessment. Clin Geriatr Med 2002;18(4):737-57.

28. Gaskill D, Black LJ, Isenring EA, Hassall S, Sanders F, Bauer JD. Malnutrition prevalence and nutrition issues in residential aged care facilities. Australas JAgeing 2008;27(4):189-94.

29. Araújo N, Britto DCC Filho, Santos FL, Costa RV, Zoccoli TLV, Novaes MRCG. Aspectos sociodemográficos, de saúde e nível de satisfação de idosos institucionalizados no Distrito Federal. Rev Ciênc Méd 2008;17(3/6):123-32.

30. Pereira MRS, Santa Cruz CMA. Risk of malnutrition among Brazilian institutionalized elderly: a study with the Mini Nutritional Assessment (MNA) questionnaire. J Nutr Health Aging 2011;15(7):532-5. 